\title{
Cherubism: Report of a Case
}

\author{
Sachin G. Ram ${ }^{1}$ Vidya Ajila² Subhas G. Babu² Pushparaja Shetty ${ }^{3}$ Shruthi Hegde ${ }^{2}$ \\ Devika S. Pillai ${ }^{4}$
}

${ }^{1}$ Dental Department, Best Hospital, Chennai, Tamil Nadu, India

${ }^{2}$ Department of Oral Medicine and Radiology, A.B. Shetty Memorial Institute of Dental Sciences, Nitte (Deemed to be University), Mangalore, India

${ }^{3}$ Department of Oral Pathology and Microbiology, A.B. Shetty Memorial Institute of Dental Sciences, Nitte (Deemed to be University),

Mangalore, India

${ }^{4}$ Dr Rajesh's Dental Lounge, Oachira, Kollam, Kerala, India
Address for correspondence Vidya Ajila, MDS, Department of Oral Medicine and Radiology, A.B. Shetty Memorial Institute of Dental Sciences, Nitte (Deemed to be University), Mangalore 575018, India (e-mail: ajila_v@yahoo.com).

J Health Allied Sci ${ }^{\mathrm{Nu}}: 2021 ; 11: 104-106$

\begin{abstract}
Keywords

- cherubism

- multilocular

- mandible

Cherubism, also known as familial fibrous dysplasia of the jaws or familial multilocular cystic disease is a rare hereditary, developmental disorder. This condition affects the posterior region of the jaws bilaterally in children belonging to the age group of 2 to 5 years. Maximum growth is recorded till puberty after which the lesion regresses over a period of time. Cherubism classically manifests radiographically as bilateral, multilocular radiolucencies affecting the posterior mandible and maxilla. Therapeutic management varies from patient to patient and is directed mainly by esthetic and functional concerns. The present report highlights the clinical and radiographic features of nonfamilial cherubism in a 6-year-old girl.
\end{abstract}

\section{Introduction}

"Familial multilocular cystic disease of jaws" was first recognized by William A. Jones in 1933. It was renamed cherubism, as the patients look like cherubs portrayed throughout Renaissance art. ${ }^{1,2}$ Though classified as a familial, autosomal dominant disease, sporadic cases have been reported in literature. ${ }^{3}$ Cherubism usually manifests at around 2 years of age, grows rapidly until puberty, and regresses by around 30 years of age. It was earlier considered to be a variant of craniofacial fibrous dysplasia localized to the jaws, but it is now confirmed as a distinct entity with a separate genetic etiology. ${ }^{4}$

The present report highlights the clinical and radiographic features of nonfamilial cherubism in a 6 -year-old girl.

\section{Case Report}

A 6-year-old girl reported to the Department of Oral Medicine and Radiology with bilateral swelling over the lower third of the face for 6 months. The swelling was gradually increasing in size and not associated with pain or limitation of mouth opening. There was no history of trauma or pus discharge. Medical, dental, and family history was noncontributory. On extraoral examination, diffuse swelling was noted over the angle of the mandible bilaterally. The skin over the swelling was normal. On palpation, swelling was nontender and bony hard in consistency with no rise in temperature (-Fig. 1A). Intraoral examination revealed erupting right mandibular first molar (-Fig. 1B). A panoramic radiograph showed multiple radiolucent areas bilaterally measuring approximately $1 \mathrm{~cm}$ in diameter distal to the permanent first molars ( - Fig. 1C). Computed tomography was advised and axial and coronal sections showed well-defined, multilocular radiolucencies bilaterally in the right and left molar region posterior to the mandibular first molars with thinning of buccal and lingual cortical plates. There were no internal calcifications within the radiolucencies ( - Fig. 2 ). Based on the clinical and radiographic features, a provisional diagnosis of cherubism published online

December 30, 2020
DOI https://doi.org/

$10.1055 / \mathrm{s}-0040-1722425$ ISSN 2582-4287.
C 2020. Nitte (Deemed to be University).

This is an open access article published by Thieme under the terms of the Creative Commons Attribution-NonDerivative-NonCommercial-License, permitting copying and reproduction so long as the original work is given appropriate credit. Contents may not be used for commercial purposes, or adapted, remixed, transformed or built upon. (https://creativecommons.org/licenses/by-nc-nd/4.0/).

Thieme Medical and Scientific Publishers Pvt. Ltd. A-12, 2nd Floor, Sector 2, Noida-201301 UP, India 

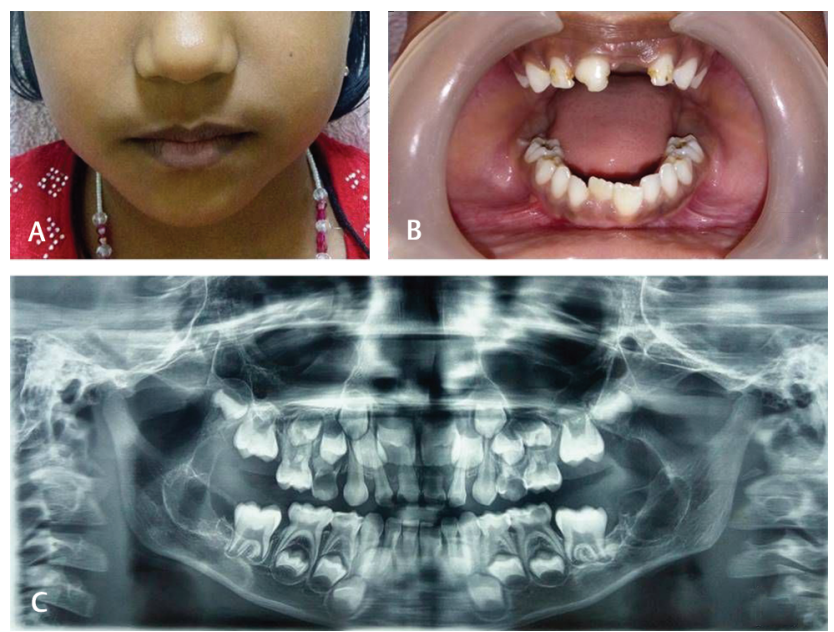

Fig. 1 (A) Diffuse extraoral swelling seen bilaterally. (B) Intraoral Photograph showing erupting right first molar and vestibular obliteration in right deciduous first and second molar region. (C) Panoramic radiograph showing bilateral unilocular radiolucency distal to the permanent molars.
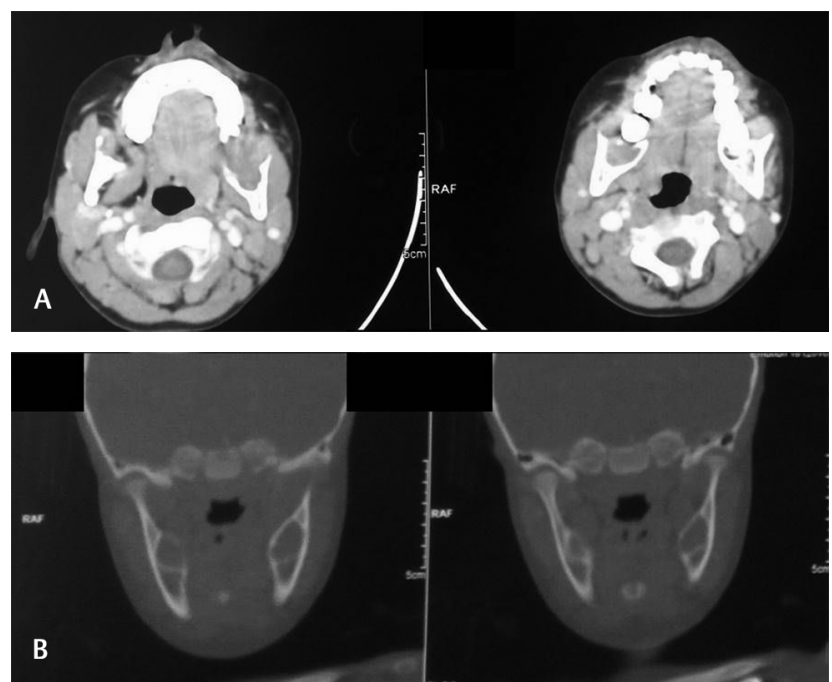

Fig. 2 (A, B) Axial and coronal sections of computed tomography showing expansion and multilocularity of the lesions.

was given. Incisional biopsy was done, and histopathology showed loosely arranged fibrocellular connective tissue with numerous multinucleated giant cells having 7 to 10 nuclei (-Fig. 3). A final diagnosis of cherubism was made based on the clinical, radiographic, and histopathological features.

\section{Discussion}

Cherubism usually manifests in the first decade around 2 years of age, following which increased growth is seen at around 8 to 9 years with spontaneous halt after puberty. Lesions usually regress completely around the third and fourth decade. ${ }^{3}$ The age at which cherubism is diagnosed depends on the severity and extent of facial deformity. ${ }^{5}$ Males are affected more than females. ${ }^{5}$

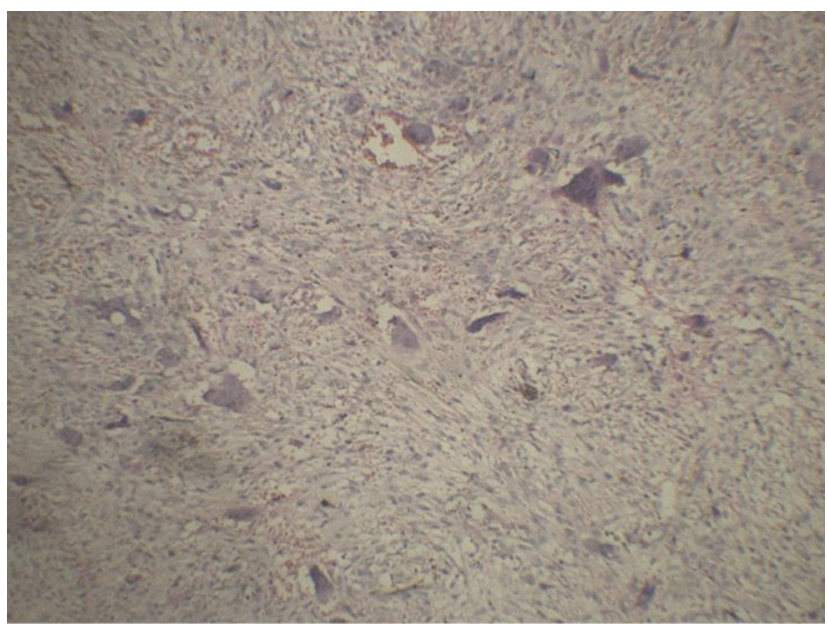

Fig. 3 Photomicrograph showing loosely arranged fibrocellular connective tissue with numerous multinucleated giant cells.

Etiopathogenesis of cherubism includes gene mutation, hormonal factors, trauma, and alteration in the mesenchyme during jaw development. Cherubism is now diagnosed as a genetic condition associated with mutation in gene SH3BP2 located on chromosome 4p16.3.,5 Mutation in the gene causes osteoclastic activation leading to the typical clinical features. The Msx-1 gene may also have a role in the etiopathogenesis of cherubism.

Cherubism is classified as quiescent, nonaggressive, and aggressive. Quiescent lesions are present in older individuals and do not demonstrate much growth. Nonaggressive lesions are seen in teenagers while the aggressive form manifests in young children with rapid growth of the lesions. ${ }^{1}$

Clinical features include painless, bilateral enlargement of the mandible and/or maxilla resulting in a rounded face along with a heavenward gaze. ${ }^{5}$ The mandible is affected more than the maxilla. ${ }^{4}$ Initial cases may present with lymph node enlargement thus contributing to the cherubic appearance., ${ }^{2,6}$ Infiltration of the orbital bone may lead to exophthalmos causing limitation of ocular movements and the typical "eyes raised to heaven" appearance. In the present case, bone involvement was restricted to the posterior mandible.

On the basis of extent of involvement, a grading system for cherubism was proposed where Grade 1 involves bilateral mandibular rami; Grade II includes bilateral mandibular rami and both maxillary tuberosities; Grade III involves the maxilla and mandible except for condylar processes and Grade IV involves the orbital floor causing orbital compression. ${ }^{7}$ The present case was classified as Grade I cherubism. The ribs, humerus, and femur have been affected in rare instances. ${ }^{8}$

Bone changes initially appear at the angle and ascending ramus of the mandible, extend to the body of the mandible and, rarely, the coronoid process. The characteristic radiographic appearance of cherubism includes bilateral, multilocular radiolucencies of the jaws without periosteal reaction. ${ }^{4}$ Displacement of mandibular canal is another feature. Associated teeth may exhibit delayed eruption, displacement, and root resorption. Missing teeth, impacted teeth, and 
floating tooth appearance of erupted teeth are other features. Involvement of the mandibular condyle is rare. ${ }^{9}$ Computed tomography and magnetic resonance imaging have been used to delineate the extent of the lesions. ${ }^{10}$ Present case showed no displacement or resorption of teeth. However, the tooth buds of the mandibular second molar were not seen.

In cases with maxillary involvement, lateral view of the skull reveals soft tissue in the posteroinferior portion of the maxillary sinus and bulging of the posterior orbital floor. ${ }^{4}$ Jain and Sharma described the "hard palate sign" where the hard palate was visible due to missing maxillary posterior teeth. ${ }^{4}$

Conditions included in the differential diagnosis of cherubism are central giant cell granuloma, giant cell tumor, fibrous dysplasia, and brown tumors of hyperparathyroidism. Central giant cell granuloma commonly involves the anterior mandible often crossing the midline while giant cell tumors rarely affect the jaw bones. Histologically, cherubism is similar to both central giant cell granuloma and hyperparathyroidism. ${ }^{5}$ Hyperparathyroidism is rare in children and can be diagnosed by analyzing parathyroid hormone, calcium, phosphorous, and alkaline phosphatase. ${ }^{11}$ Cherubism may be associated with syndromes such as neurofibromatosis type I, Noonanlike/multiple giant cell lesion syndrome, Ramon syndrome, and Jaffe-Campanacci syndrome. ${ }^{12}$ Bilaterally symmetrical, multilocular radiolucent lesions in the posterior mandible and maxilla in a young patient with familial history suggest a diagnosis of cherubism.

Cherubism has been associated with a central odontogenic fibroma-like proliferation. Argyris et $\mathrm{al}^{6}$ reported the development of intraosseous squamous cell carcinoma in a long-standing case of cherubism. Osteosarcoma has been reported in cherubism cases treated with radiotherapy.

Management of cherubism first includes extraction of associated impacted teeth. Since the disease tends to regress with age, a wait and watch approach can be used in a majority of the cases as in the present case. Surgical correction can be done if needed at a later date especially in patients who are concerned about the facial deformity. Aggressive lesions with marked facial deformity need early surgical intervention for corrective and esthetic purpose.,13

\section{Conclusion}

Cherubism is a rare childhood disease with distinct clinical and radiographic features. Clinicians must always consider the possibility of cherubism in a young subject with bilateral, multilocular, radiolucent lesions of the jaws thereby preventing unnecessary surgical intervention. Since this condition regresses with age, treatment should be tailored according to esthetic and functional concerns.

\section{Authors' Contributions}

All authors contributed to conception and design, drafting, and critical revision of the article.

\section{Conflict of Interest}

None declared.

\section{References}

1 Mani S, Natarajan B, Rajaram K, Sahuthullah YA, Gokulanathan S, Sitra G. Rare form of cherubism: case report with review of literature. J Pharm Bioallied Sci 2013;(suppl 2):S142-S146

2 Papadaki ME, Lietman SA, Levine MA, Olsen BR, Kaban LB, Reichenberger EJ. Cherubism: best clinical practice. Orphanet J Rare Dis 2012;7(suppl 1):S6

3 Mehrotra D, Kesarwani A, Nandlal. Cherubism: case report with review of literature. J Maxillofac Oral Surg 2011;1 $0(1): 64-70$

4 Jain V, Sharma R. Radiographic, CT and MRI features of cherubism. Pediatr Radiol 2006;3 6(10):1099-1104

5 Lima Gde M, Almeida JD, Cabral LA. Cherubism: clinicoradiographic features and treatment. J Oral Maxillofac Res 2010;1( 2):e2

6 Argyris PP, Gopalakrishnan R, Hu Y, Reichenberger EJ, Koutlas IG. Clinicopathologic and molecular characteristics of familial cherubism with associated odontogenic tumorous proliferations. Head Neck Pathol 2018;12(1):136-144

7 Ramon Y, Engelberg IS. An unusually extensive case of cherubism. J Oral Maxillofac Surg 1986;44(4):325-328

8 Davis GB, Sinn DP, Watson SW. Case 43, part II: Cherubism. J Oral Maxillofac Surg 1983;41(2):119-120

9 Agrawal A, Gupta SK, Saxena P, Kumar P. Non-familial cherubism: clinical and radiological findings. BMJ Case Rep 2014;2 014:bcr2013202031

10 Elshafey R. Imaging of cherubism: case report and review of the literature. Tanta Med J 2014;42(1):42-45

11 Papadaki ME, Lietman SA, Levine MA, Olsen BR, Kaban LB, Reichenberger EJ. Cherubism: best clinical practice. Orphanet J Rare Dis 2012;71(suppl 1):S6

12 Deshmukh R, Joshi S, Deo PN. Nonfamilial cherubism: a case report and review of literature. J Oral Maxillofac Pathol 2017;21(1):181

13 Kheir MK. Case report of nonfamilial cherubism in a toddler: description of clinic-radiographic features and osseous-dental treatments. Case Rep Med 2016;(9):1-5. Doi:https://doi. org/10.1155/2016/8795765 